\title{
Handling of Virtual Cloth
}

\author{
Shigeru Inui, Yuko Mesuda, and Yosuke Horiba \\ ShinshuUniversity, Faculty of Textile Science and Technology, Tokida. 3-15-1, Ueda, \\ Nagano, 386-8567, Japan \\ \{inui, 12st115c, horiba\} ashinshu-u.ac.jp
}

\begin{abstract}
In this study, manipulations of virtual cloth model were examined for draping. Cloth was mechanically formalized and modeled. Motion of real human hand is detected by a sensor and the motion is utilized for handling of the cloth model. Cloth manipulations to hold, release and attach were realized. When the cloth model is handling, "coarse model" and fast dynamic calculation method is used and when the cloth is at rest, "precise model" and precise static calculation method is used. When the cloth model is stopped from dynamic regime, the model of the cloth is switched from "coarse" to "precise". When the model is started to move from static regime, the model of the cloth is switched from "precise" to "coarse".
\end{abstract}

Keywords: virtual draping, real-time, hand gesture, cloth manipulation.

\section{Introduction}

Draping is one of a design method for clothing. Draping is a method to design paper patterns by putting cloth on dummy surface and forming the shape of clothing. Though draping has many advantages such as it is possible to make clothing fit for each person, it costs much time and money. As it is possible to decrease the costs drastically by virtualizing draping processes, some methods of virtual draping are proposed. Real time cloth handling is important for virtual draping. Some methods have been proposed[1-3], and mouse is utilized for handling of virtual cloth. The method of handling virtual cloth by the motion of real human hands is more realistic than by mouse manipulation. We examined handling methods by the motion of real human hands.

\section{Model and Sensor}

\subsection{The Cloth Model and the Object Model}

In our study, the virtual cloth model for handling consists of particles and springs. The particles are aligned in a reticular pattern, and neighboring particles are connected by crosswise, longitudinal and diagonal spring. Other rigid virtual objects consist of particles but the particles are not connected by spring and the objects are not deformed. 


\subsection{Dynamic Calculation}

Inner force in the cloth, collision force with other objects and the gravity force act on each particle of the cloth model and the forces are calculated. The forces are summed, the summed up forces are integrated by leap-frog method and each position of the particle in the next step is obtained [4].

\subsection{Collision Reaction and Collision Detection.}

To calculate collision force, collision between cloth model and virtual object has to be detected. When the distance between cloth model and other virtual object is less than predefined threshold, collision is detected, and then collision force acts on the repulsive colliding particle in the cloth model.

\section{Manipulation of Cloth Model}

In this study, the virtual cloth model is handled by motion of real human hand. Motion of human hand in the real world is detected by a sensor, and Kinct is used for the sensor. In Kinect, a camera and sensors are equipped and three-dimensional coordinate of the joints of human body can be acquired. The coordinates of joints are updated every one-thirty second and motion of human body can be acquired. Here, some fundamental manipulation of cloth for draping are realized with the acquired human body motion.

\subsection{Manipulation to Hold and Release Cloth Model}

The manipulation to hold cloth model is realized to move one or two particles in the cloth model coordinating the movement of real human hands. The position of the particles is constrained to the movement of real human hands. To release the cloth model, the restriction between the particles and the motion of real human hands is canceled. The cancellation is triggered by a key operation.

\subsection{Manipulation to Attach Cloth Model}

The manipulation to attach the cloth model is to put handling cloth model on a virtual object. The manipulation to attach the cloth model is triggered by a key operation, some nodes in the cloth model are fixed on the nearest virtual object and the restriction between the particles and the motion of real human hands is canceled. When the cloth model is attached to a virtual object, collision between the cloth model and the object is detected and collision reaction is processed. 


\section{3 "Coarse" or "Precise" Model}

To manipulate virtual cloth in real time, it is desirable that the cloth model is simple as much as possible. On the other hand, to predict the precise shape of real cloth by simulation, it is desirable that the cloth model is detailed model as much as possible. The cloth model is a trade-off between response and precision. For this reason, a mechanism is introduced in which a simple cloth model is applied when the model is manipulated and a precise model is applied when the model is at rest.

A coarse mesh is utilized for the cloth model when cloth model is handled. Cloth is modeled by mass and spring model, and the shape of virtual cloth is calculated by integration of motion equation. The model is called "coarse model". A precise mesh is applied for the cloth model when the virtual mesh is not handled. Cloth is modeled as continuum, and the shape of the virtual cloth is calculated by minimization of energy of the model. The model is called "precise model".

\subsection{Switching Models}

While the cloth model is handled, the position of the hands of operator is detected by sensor, and the coordinate of the nodes of the cloth model is updated according to the movement of the hands of the operator. The handling of the virtual cloth model by real human hands is realized by the technique. The "coarse model" is applied in the situation above. Handling of virtual cloth is finished when some nodes of the model are fixed or the model is laid on other virtual object. When handling is finished, the model of the virtual cloth is switched from "coarse model" to "precise model". The shape of the "precise model" is obtained from the "coarse model", and the precise calculation is started from the shape as the initial shape. On the other hand, when the virtual cloth is at rest, the "precise model" is applied. When handling of the virtual cloth is started, the cloth model is switched from the "precise model" to the "coarse model" for real time calculation. When the cloth model is attached to a virtual object, collision between the cloth model and the object is detected and collision reaction is processed.

\section{$4 \quad$ Result and Discussion}

Manipulation to hold or release was started from the initial condition in which one of the nodes or both nodes of upper end of the cloth model were fixed. It is possible to hold any point of the virtual cloth model, and after that the shape of the cloth model is changed coordinating the motion of real human hands. It is confirmed that the cloth model falls off after cancellation of the restriction between two nodes or one node at the edge of the cloth model and the motion of real human hands shown in figure 1. It is also confirmed that the cloth model is attached to a virtual object. Some gap was observed between the cloth and the object model. The gap was decreased to increase iterations of mechanical and collision calculation.

The "coarse model" is the model of lattice-shaped mesh model. To make the "precise model" from the "coarse model", edge of the unit lattice is divided and new 
lattice nodes are created in every unit lattice by proportional division. The "coarse model" of the virtual cloth is shown in the left side of figure 2, and the "precise model" made from the "coarse model" is shown in the right side of figure 2. The "precise model" is a triangular mesh divided from a lattice-shaped mesh. The "coarse model" is made by picking up for example every three nodes form the nodes of the lattice of the "precise model".
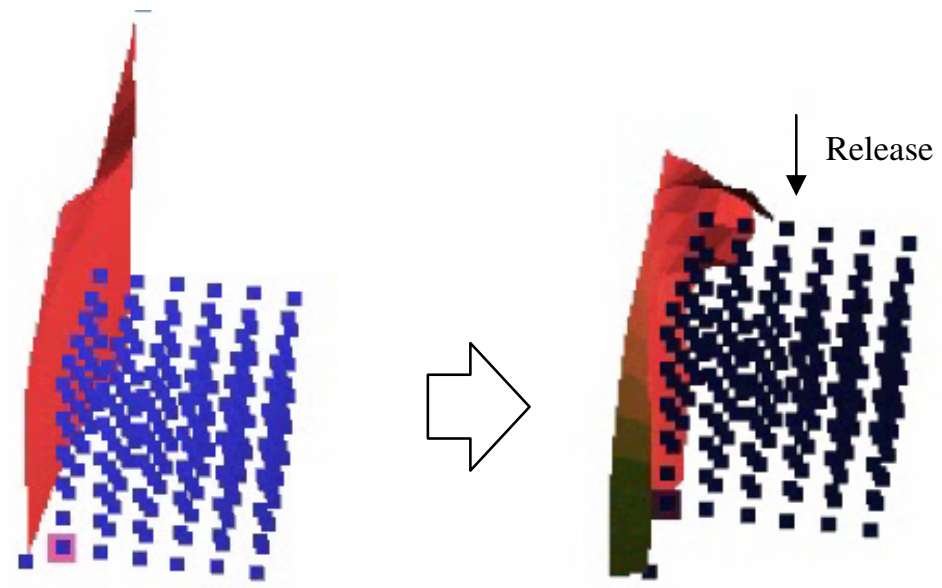

Fig. 1. The small cubes are the nodes of a virtual object. The left side shows that virtual cloth is holding by two corner nodes and the right side shows that one of the nodes is released.
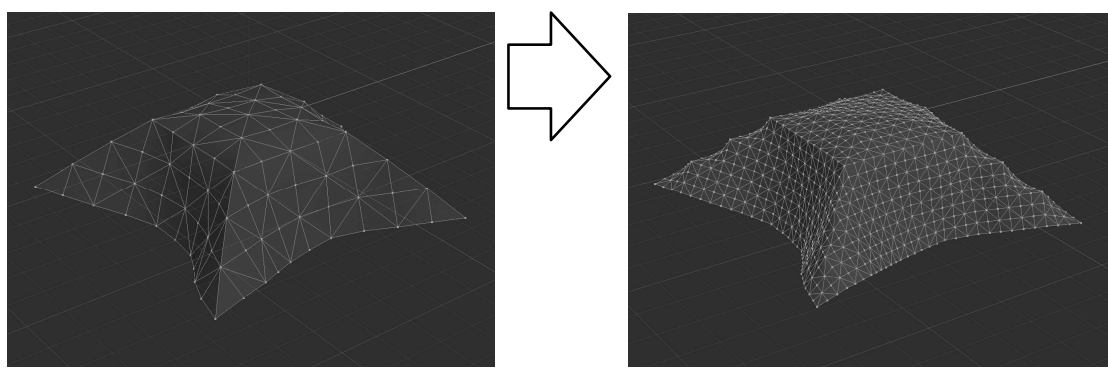

Fig. 2. The mesh of the left side is 10 by 10 lattice structure of "coarse" model. The mesh of the right side is 30 by 30 lattice structure of "precise" model made from the "coarse" model.

\section{Conclusion}

In this study, manipulations of virtual cloth model were examined for draping. Cloth manipulations to hold, release and attach were realized. It is necessary to extract other manipulations for draping, and other manipulations such as to fold or cut the cloth model should be realized. Then, it is expected to construct a draping system combining those manipulations. 
Acknowledgments. This work was partly supported by Grants-in-Aid for Scientific Research (No. 23240100, 24220012) from the Ministry of Education, Science, Sports and Culture and Grants for Excellent Graduate Schools, MEXT, Japan.

\section{References}

1. Meng, Y., Mok, P.Y., Jin, X.: Interactive Virtual Try-on Clothing Design Systems. Computer-Aided Design 147, 310-321 (2010)

2. Igarashi, T., Hughes, J.F.: Clothing Manipulation. In: UIST and I3D Reprise session, ACM SIGGRAPH, p. 697 (2003)

3. Mesuda, Y., Inui, S., Horiba, Y.: Cutting Method for Cloth Handling. In: Proceedings of 1st International Symposium on Affective Engineering, pp. 145-151 (2013)

4. Harada, T., Koshizuka, S.: Real-time Collision Computation Between Cloth and Highlytessellated Models. Transaction of Information Processing Society of Japan 48, 1829-1837 (2007) 